\title{
Do more attractive women show stronger preferences for male facial masculinity?
}

Ciaran Docherty ${ }^{1}$, Anthony J Lee ${ }^{2}$, Amanda C Hahn, Lisa M DeBruine, Benedict C Jones ${ }^{1}$

1. Institute of Neuroscience \& Psychology, University of Glasgow, Scotland

2. Faculty of Natural Science, Division of Psychology, University of Stirling, Scotland

3. Department of Psychology, Humboldt State University, USA

Data and analysis code publicly available at https://osf.io/36fs5/

The authors thank Iris Holzleitner for helpful discussions about these data and code check 


\section{Do more attractive women show stronger preferences for male facial masculinity?}

\section{Abstract}

Researchers have suggested that more attractive women will show stronger preferences for masculine men because such women are better placed to offset the potential costs of choosing a masculine mate. However, evidence for correlations between measures of women's own attractiveness and preferences for masculine men is mixed. Moreover, the samples used to test this hypothesis are typically relatively small. Consequently, we conducted two large-scale studies that investigated possible associations between women's preferences for facial masculinity and their own attractiveness as assessed from third-party ratings of their facial attractiveness (Study $1, N=454$, laboratory study) and self-rated attractiveness (Study 2, N = 8972, online study). Own attractiveness was positively correlated with preferences for masculine men in Study 2 (self-rated attractiveness), but not Study 1 (thirdparty ratings of facial attractiveness). This pattern of results is consistent with the proposal that women's beliefs about their own attractiveness, rather than their physical condition per se, underpins attractiveness-contingent masculinity preferences.

\section{Introduction}

Trade-off theories of women's preferences for masculine men propose that men displaying more masculine physical characteristics are more likely to be healthy, physically strong, and able to compete for resources, but also less likely to invest time and effort in their mates and offspring (Little et al., 2001; Penton-Voak et al., 2003). According to such theories, women may then differ systematically in how they weigh up the costs and benefits of choosing a masculine mate (Little et al., 2001; Penton-Voak et al., 2003).

One factor that is widely thought to influence how women resolve this trade off between the potential costs and benefits of choosing a masculine mate is women's own physical attractiveness (Little et al., 2001; Penton-Voak et al., 2003). Specifically, more attractive women are predicted to prefer more 
masculine mates. The rationale for predicting such attractiveness-contingent masculinity preferences is that more physically attractive women will be better able to retain and secure investment from masculine men and/or better able to replace masculine men in the event of relationship dissolution (Little et al., 2001; Penton- Voak et al., 2003). In other words, more attractive women are more likely to prefer masculine men as mates because more attractive women are better able to minimize the potential costs of choosing a masculine mate, such as low investment and increased risk of desertion.

Several studies have investigated possible correlations between women's own attractiveness and their preferences for facial masculinity by measuring women's own attractiveness via self-ratings. However, results from studies using this methodology are mixed. Little et al. (2001, $N=66)$, Kandrik and DeBruine (2012, $N=1000$ ), and Batres et al. (in press, $N=27611$ ) found that women who rated their own attractiveness higher showed stronger preferences for men with masculine facial characteristics. Relatedly, Vukovic et al. $(2008, N=58)$ found that women who rated their own attractiveness higher showed stronger preferences for masculine (i.e., low) pitch in men's voices. By contrast, neither Penton-Voak et al. $(2003, \mathrm{~N}=36)$ nor Zietsch et al. (2015, N = 2160) observed significant positive correlations between women's self-rated attractiveness and facial masculinity preferences.

Other work has tested for evidence that women's own physical attractiveness predicts the strength of their masculinity preferences by assessing women's own attractiveness via third-party attractiveness ratings of face photographs. Using this methodology, Penton-Voak et al. $(2003, N=35)$ found that more attractive women showed stronger preferences for men with masculine facial characteristics when assessing men's attractiveness for hypothetical longterm, but not short-term, relationships. This result was interpreted as strong evidence for trade-off theories of women's preferences for masculine men because the potential costs of choosing a masculine mate are generally assumed to be more pronounced for long-term, than short-term, relationships (Penton-Voak et al., 2003). Consistent with Penton-Voak et al's results, Smith et al. $(2009, N=42)$ found that women with lower (i.e., more attractive) waist- 
to-hip ratios showed stronger preferences for men's facial masculinity and O'Connor et al. $(2012, \mathrm{~N}=63)$ found that women whose faces were rated more attractive showed stronger preferences for men's facial and vocal masculinity.

With only three exceptions (Batres et al., in press; Kandrik \& DeBruine, 2012; Zietsch et al., 2015), the studies described above testing for correlations between women's masculinity preferences and own attractiveness tested small samples of women. Moreover, there has not yet been a large-scale replication attempt of the finding that any objective (i.e., not self report) measure of women's attractiveness predicts variation in masculinity preferences. Consequently, we conducted two large-scale studies of women's attractiveness-contingent masculinity preferences.

Study 1 (laboratory study) was a direct replication of Penton-Voak et al. (2003) and investigated the possible relationship between third-party ratings of facial attractiveness and preferences for masculinity in men's faces in a sample of 454 women. Study 2 (online study) investigated possible relationships between self-ratings of attractiveness and preferences for masculine characteristics in men's and women's faces in a sample of 8972 women.

\section{Study 1}

\section{Methods}

\section{Participants}

Participants were 454 heterosexual women (mean age $=21.43$ years, $S D=$ 3.18 years), participating as part of a longitudinal study of potential hormonal correlates of women's face preferences and own facial appearance (Jones et al., 2018). To match Penton-Voak et al's (2003) study design, only face preferences and face images from each participant's first test session were analyzed in the current study. Women in Study 1 participated in the lab.

Simonsohn (2015) recommends that sample sizes for replication studies be at least two-and-a-half times larger than the sample size in the original study. 
The total sample size in Penton-Voak et al. (2003) was 82 women and the critical correlation between facial attractiveness and long-term masculinity preferences was based on an analysis of responses from 35 women. Thus, our sample exceeds the size that Simonsohn (2015) recommends for replications.

The order in which women completed the face preference tests and had their face photograph taken was fully randomized. All participants were staff or students at the University of Glasgow.

\section{Assessing women's preferences for facial masculinity}

The methods we used to manufacture stimuli to test women's preferences for facial masculinity have been used in many previous studies, including PentonVoak et al. (2003). Stimuli from this study are publicly available at www.osf.io/ 9b4y7. Briefly, we first manufactured a female prototype (i.e., average) face by using specialist software (DeBruine, 2018; Tiddeman et al., 2001) to average the shape, color, and texture information from images of 50 young white women's faces. A male prototype face was also manufactured in this way by averaging the shape, color, and texture information from images of 50 young white men's faces. Next, we randomly selected 10 images from the set of 50 individual male faces. We then created a feminized and a masculinized version of each of these 10 male images by adding or subtracting $50 \%$ of the linear (i.e., vector) differences in 2D shape between symmetrized versions of the female and male prototypes to (or from) each individual image. This process created 10 pairs of face images in total, with each pair consisting of a feminized and a masculinized version of one of the individual face images.

Examples of these stimuli are shown in Figure 1. Note that our feminized and masculinized versions of faces differed in sexually dimorphic shape characteristics only (i.e., were matched in other regards, such as identity, color, and texture, Tiddeman et al., 2001). 

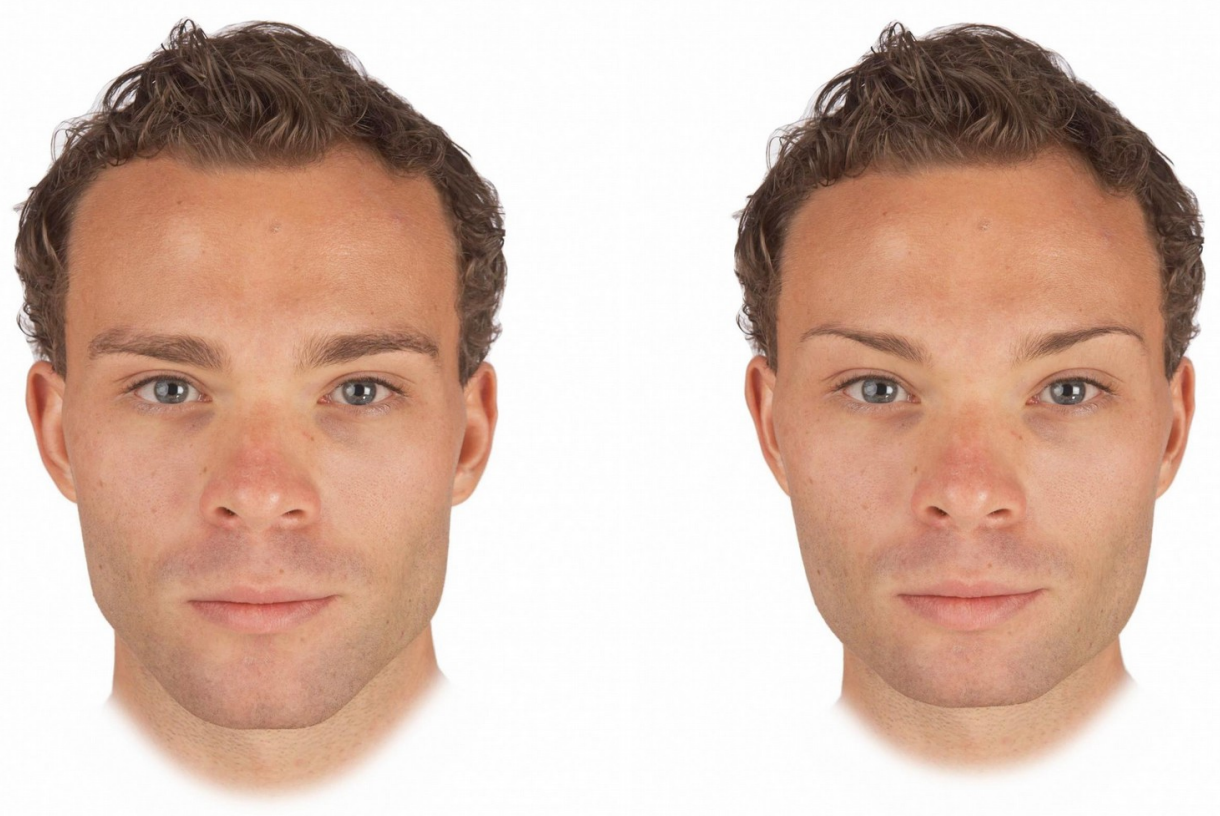

Figure 1. Examples of masculinized (left) and feminized (right) versions of men's faces used to assess facial masculinity preferences in our study.

Each participant completed two face preference tests (one assessing men's attractiveness for a short-term relationship, the other assessing men's attractiveness for a long-term relationship). Trial order within each test was fully randomized and the order in which the two face preference tests were completed was also fully randomized.

In the two face preference tests, women were shown the 10 pairs of male faces, each pair consisting of a masculinized and feminized version of a given individual. Women were instructed to select the more attractive face in each pair and to indicate the strength of that preference by choosing from the options "slightly more attractive", "somewhat more attractive", more attractive", and "much more attractive". This procedure has been used to assess masculinity preferences in previous studies (e.g., Zietsch et al., 2015). In each face preference test, the 10 trials assessing preferences for sexually dimorphic shape characteristics were interspersed among 30 filler trials assessing preferences for other facial traits. Definitions of short-term and long-term relationships were identical to those used in Penton-Voak et al. 
(2003). Face preferences were collected using the Experimentum interface (DeBruine, 2019).

In the short-term attractiveness test, women were told: "You are looking for the type of person who would be attractive in a short-term relationship. This implies that the relationship may not last a long time. Examples of this type of relationship would include a single date accepted on the spur of the moment, an affair within a long-term relationship, and possibility of a one-night stand."

In the long-term attractiveness test, women were told: "You are looking for the type of person who would be attractive in a long-term relationship. Examples of this type of relationship would include someone you may want to move in with, someone you may consider leaving a current partner to be with, and someone you may, at some point, wish to marry (or enter into a relationship on similar grounds as marriage)."

Responses on the face preference test were coded using the following scale (higher scores indicate stronger masculinity preferences and the scale is centered on chance, i.e., zero):

0.5 to 3.5: masculinized face rated 'slightly more attractive' $(=0.5)$, 'somewhat more attractive' $(=1.5)$, 'more attractive' $(=2.5)$ or 'much more attractive' $(=3.5)$ than feminized face.

-0.5 to -3.5 : feminized face rated 'slightly more attractive' $(=-0.5)$, 'somewhat more attractive' (=-1.5), 'more attractive' (=-2.5) or 'much more attractive' (=3.5) than masculinized face.

\section{Assessing other-rated facial attractiveness}

Ratings of women's facial attractiveness were collected and analyzed previously in Holzleitner et al. (in press). Each woman first cleaned her face with hypoallergenic face wipes to remove any make up. A full-face digital photograph was taken a minimum of 10 minutes later. Photographs were taken in a small windowless room against a constant background, under 
standardized diffuse lighting conditions, and participants were instructed to pose with a neutral expression. Camera-to-head distance and camera settings were held constant. Participants wore a white smock covering their clothing when photographed to control for possible effects of reflectance from clothing. Photographs were taken using a Nikon D300S digital camera with an AF Micro-Nikkor $60 \mathrm{~mm}$ f/2.8D lens. A GretagMacbeth 24-square ColorChecker chart was included in each image for use in color calibration. Images were color-calibrated using a least-squares transform from an 11expression polynomial expansion developed to standardize color information across images (Hong et al., 2001). Each image was masked so that hairstyle and clothing were not visible, placed on a black background, and standardized on pupil positions prior to rating.

The face images were then rated for attractiveness using a 1 (much less attractive than average) to 7 (much more attractive than average) scale by 16 men and 16 women. Trial order was fully randomized. Inter-rater agreement was high for these ratings and ratings by male and female raters were highly correlated (see Holzleitner et al., in press). Consequently we calculated an average attractiveness score for each image to use in our analyses.

\section{Results}

Masculinity preferences were analyzed using linear mixed effects models using the Ime4 (Bates et al., 2015) and ImerTest (Kuznetsova et al., 2015) packages in the $R$ statistical software (R Core Team, 2013). Predictors were facial attractiveness, which was z-standardized, and relationship context, which was effect coded (-.5 = short-term context, $.5=$ long-term context). Random intercepts were specified for participants and stimuli and random slopes were specified maximally (Barr et al., 2013; Barr, 2013). The model included main effects of facial attractiveness and relationship context, as well as the interaction between facial attractiveness and relationship context. Full analysis code and results can be seen at https://osf.io/36fs5/. 


\begin{tabular}{rrrr}
\hline & $\begin{array}{r}\text { Estimate } \\
\text { (Std Error) }\end{array}$ & $\begin{array}{r}\text { t-value } \\
\text { (approx df.) }\end{array}$ & \\
\hline Intercept & $.38(.13)$ & $2.88(9.52)$ & $.017^{*}$ \\
Relationship Context & $-.04(.02)$ & $-2.11(63.98)$ & $.039^{*}$ \\
Facial Attractiveness & $.02(.02)$ & $.75(363.63)$ & 0.456 \\
Relationship Context * Facial Attractiveness & $-.02(.02)$ & $-1.16(80.55)$ & 0.249 \\
\hline
\end{tabular}

Table 1. Summary of results of masculinity-preference analysis (Study 1).

Results are summarized in Table 1 and Figure 2. The intercept was significant and positive (estimate $=0.37880, \mathrm{SE}=0.13162, \mathrm{df}=9.52424, \mathrm{t}=2.878, \mathrm{p}=$ 0.0172 ), indicating that women generally preferred masculinized versions of men's faces to feminized versions. The main effect of relationship context was also significant and positive (estimate $=-0.03757, \mathrm{SE}=0.01785, \mathrm{df}=$ $63.98154, t=-2.105, p=0.0392$ ), indicating that women's masculinity preferences were generally stronger for short-term than long-term relationships. Neither the main effect of facial attractiveness (estimate $=$ $0.01728, \mathrm{SE}=0.02318, \mathrm{df}=363.62765, \mathrm{t}=0.745, \mathrm{p}=0.4565)$ nor the interaction between facial attractiveness and relationship context (estimate $=$ 0.02017, $\mathrm{SE}=0.01736, \mathrm{df}=80.55434, \mathrm{t}=-1.162, \mathrm{p}=0.2486$ ) were significant, indicating that women's own attractiveness did not predict masculinity preferences. 


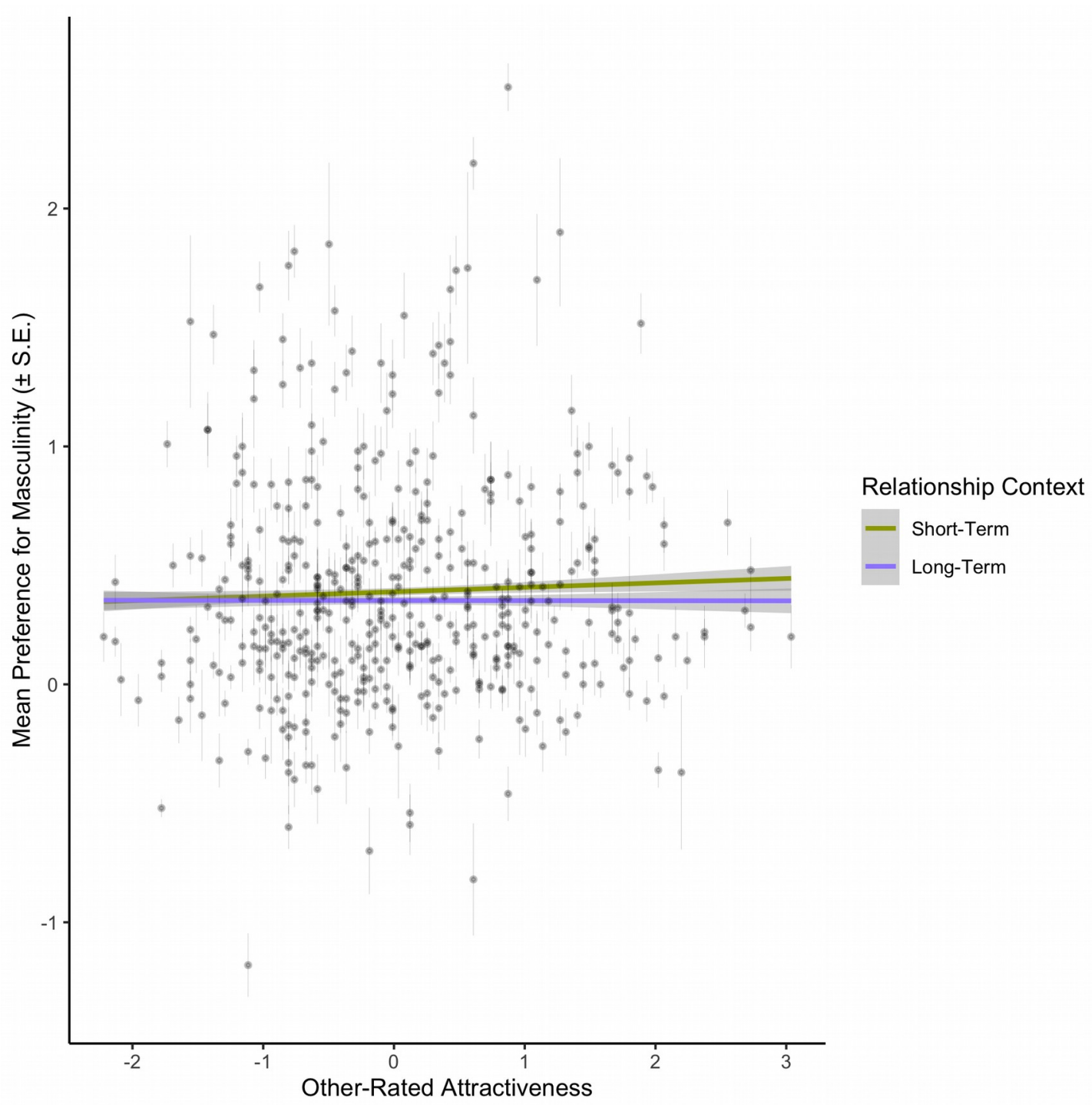

Figure 2. The relationship between facial attractiveness and women's masculinity preferences in short- and long-term contexts (Study 1).

\section{Study 2}

\section{Methods}

8972 heterosexual women (mean age $=22.9$ years, $S D=5.46$ years) took part in the study. The study was run online at faceresearch.org.

For Study 2, 50\% of the linear differences in 2D shape between symmetrized versions of the male and female prototypes were added to or subtracted from face images of 20 young adult White men and 20 young adult White women. Forty pairs of images were produced in total (20 pairs of female face images and 20 pairs of male face images) with each pair consisting of a masculinized 
and a feminized version of the same individual. Participants were shown the 40 pairs of face images and were asked to choose the face in each pair that was more attractive. Participants also indicated the strength of these preferences by choosing from the options 'slightly more attractive', 'somewhat more attractive, 'more attractive', and 'much more attractive'. The order in which pairs of faces were shown was fully randomized for each participant and the side of the screen on which any particular image was shown was also fully randomized. The stimuli used in Study 2 are publicly available (DeBruine \& Jones, 2017). Face preferences were collected using the Experimentum interface (DeBruine, 2019).

Responses on the face preference test were coded using the following scale (higher scores indicate stronger masculinity preferences and the scale is centered on chance, i.e., zero):

0.5 to 3.5: masculinized face rated 'slightly more attractive' (=0.5), 'somewhat more attractive' (=1.5), 'more attractive' (=2.5) or 'much more attractive' (=3.5) than feminized face.

-0.5 to -3.5 : feminized face rated 'slightly more attractive' (=-0.5), 'somewhat more attractive' (=-1.5), 'more attractive' (=-2.5) or 'much more attractive' (=3.5) than masculinized face.

Participants also rated their own attractiveness using a 1 (much less attractive than average) to 7 (much more attractive than average) scale. The order in which participants completed the face preference test and rated their own attractiveness was fully randomized.

\section{Results}

All data and analysis code can be found at https://osf.io/36fs5/. Preference scores were analyzed using linear mixed effects models using the Ime4 (Bates et al., 2015) and ImerTest (Kuznetsova et al., 2015) packages in $\mathrm{R}$ statistical software (R Core Team, 2013). The model included fixed effects for sex of face and self-rated attractiveness, as well as their interaction. Sex of 
face was effect coded (male $=0.5$, female $=-0.5$ ) and self-rated attractiveness was centred on the mean for the sample. Random intercepts were specified for both participants and stimuli and random slopes were specified maximally (Barr et al., 2013; Barr, 2013).

\begin{tabular}{rllr}
\hline & $\begin{array}{l}\text { Estimate } \\
\text { (Std Error) }\end{array}$ & \multicolumn{1}{l}{ t-value } \\
(approx df.) & \multicolumn{1}{c}{ p-value } \\
\hline Intercept & $-.50(.07)$ & $-7.15(38.36)$ & $<.001^{* * *}$ \\
Face Sex & $.96(.14)$ & $6.85(38.27)$ & $<.001^{* * *}$ \\
Self-Rated Attractiveness & $-.01(.01)$ & $-1.44(182.94)$ & 0.151 \\
Face Sex * Self-Rated Attractiveness & $.10(.01)$ & $8.34(136.38)$ & $<.001^{* * *}$ \\
\hline
\end{tabular}

Table 2. Summary of results of femininity-preference analysis (Study 2).

The main effect of face sex (estimate $=0.96, \mathrm{SE}=0.14, \mathrm{t}(38.3)=6.85, \mathrm{p}$ $<.001)$ meant that masculinity preferences were stronger for male faces than for female faces. There was no significant main effect of SRA (estimate = $0.01, \mathrm{SE}=0.01, \mathrm{t}(182.9)=-1.44, \mathrm{p}=0.151)$. The interaction between SRA and face sex (estimate $=0.10, \mathrm{SE}=0.01, \mathrm{t}(136.4)=8.34, \mathrm{p}<.001)$ meant that masculinity preferences decreased with increasing SRA for female faces (estimate $=-0.06, \mathrm{SE}=0.01, \mathrm{t}(128.0)=-8.05, \mathrm{p}<.001)$, but increased with increasing SRA for male faces (estimate $=0.04, \mathrm{SE}=0.01, \mathrm{t}(66.1)=4.06, \mathrm{p}<$ .001). These results are summarized in Figure 3 and Table 2. 


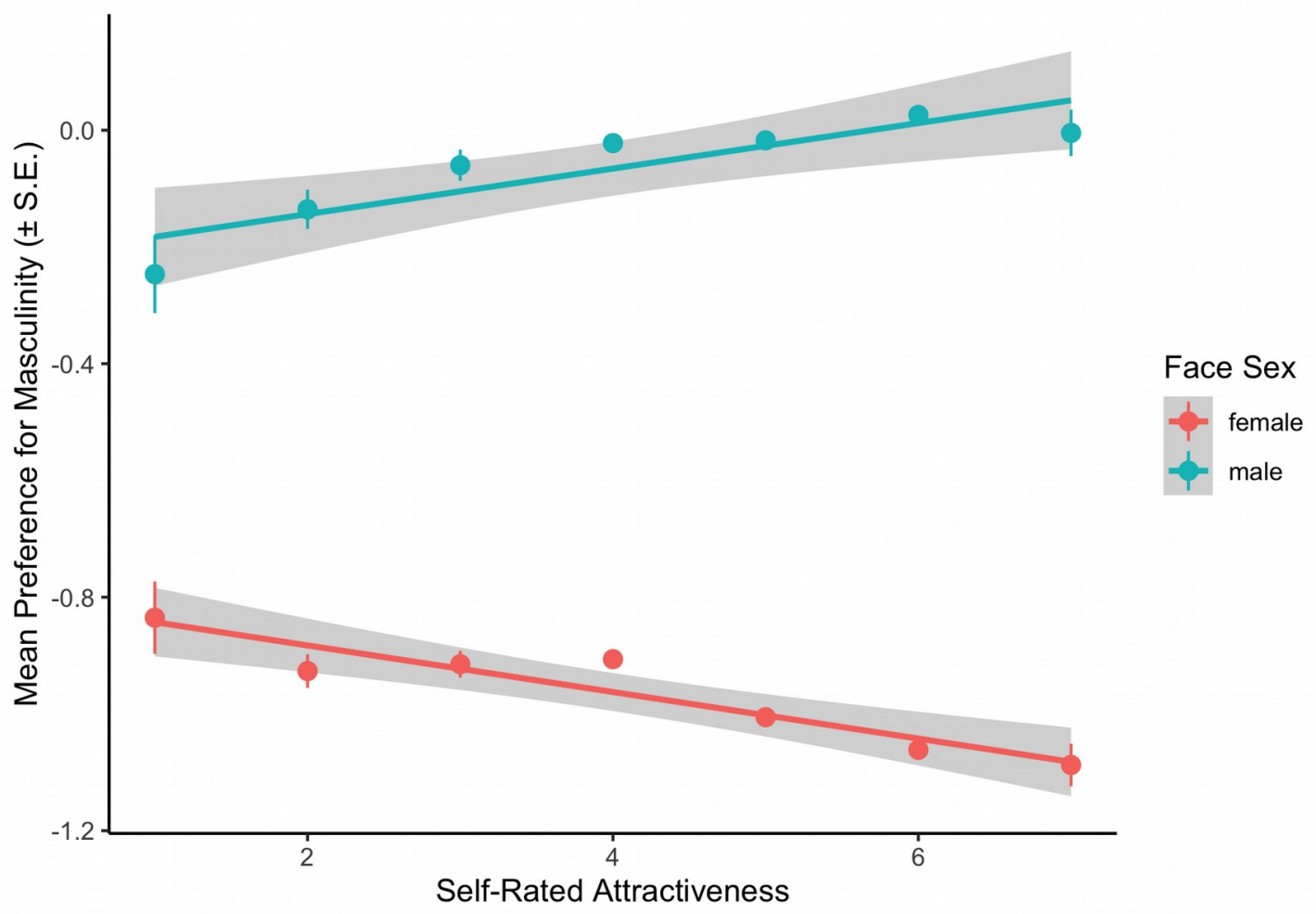

Figure 3. The relationships between self-rated attractiveness and women's preferences for masculinity in men's and women's faces (Study 2).

\section{Discussion}

Study 1 did not find any compelling evidence that third-party ratings of women's facial attractiveness modulated the strength of their facial masculinity preferences (see Figure 2). Thus, we did not replicate results of previous studies in which women judged to have more attractive faces showed stronger preferences for masculine men (Penton-Voak et al., 2003; O'Connor et al., 2012). However, in Study 1, we did find that women showed slightly stronger preferences for masculine men for hypothetical short-term relationships. This result is then consistent with the proposal that the antisocial traits masculine men are perceived to have are unattractive in longterm partners and/or that perceived traits like dominance that are reliably associated with facial masculinity in men are particularly attractive in shortterm relationships (Little et al., 2002; Penton-Voak et al., 2003).

Study 2 found weak, but significant correlations, between women's self-rated attractiveness and masculinity preferences (see Figure 3). Women who rated 
themselves as more attractive showed stronger preferences for masculinity in men's faces, but also showed stronger preferences for femininity in women's faces. The results for male faces replicate those reported in some previous studies (Batres et al., in press; Little et al., 2001; Kandrik \& DeBruine, 2012). The results for female faces suggest that the effects of self-rated attractiveness on women's preferences for exaggerated sex-typical facial characteristics are not specific to judgments of opposite-sex faces, but also may extend to judgments of own-sex faces.

Collectively, our results suggest that women's self-rated attractiveness may be a better predictor of their preferences for masculinity in men's faces than are third-party ratings of their facial attractiveness. Little and Mannion (2006) have previously reported that experimentally increasing women's perceptions of their own attractiveness by exposing them to images of low-attractive women increased their preferences for masculinity in men's faces. They interpreted this result as suggesting that women calibrate their own attractiveness according to the attractiveness of other women and calibrate their masculinity preferences according to perceptions of their own attractiveness, rather than calibrating them according to their own physical condition. That we found women's masculinity preferences were related to their self-rated attractiveness, but not third-party ratings of their facial attractiveness, is consistent with Little and Mannion's (2006) proposal.

It is potentially important to note that we investigated only two measures of women's own attractiveness (self-ratings and third-party ratings of faces) and investigated also only investigated face preferences. Our results suggest that previous findings for self-rated attractiveness, but not third-party attractiveness-ratings of faces, predict individual differences in preferences for facial masculinity. However, further work investigating other measures of women's own attractiveness (e.g., body shape or adiposity) and preferences for masculine characteristics in men's faces, bodies, and behaviors may further clarify the issue of when and how women's own attractiveness predicts their masculinity preferences. 


\section{References}

Barr, D. J. (2013). Random effects structure for testing interactions in linear mixed-effects models. Frontiers in Psychology, 4, 328.

Barr, D. J., Levy, R., Scheepers, C., \& Tily, H. J. (2013). Random effects structure for confirmatory hypothesis testing: Keep it maximal. Journal of Memory and Language, 68(3), 255-278.

Bates, D., Mächler, M., Bolker, B. M., \& Walker, S. C. (2015). Fitting linear mixed-effects models usng Ime4. Journal of Statistical Software, 67(1), 1-48.

Batres, C., Jones, B. C., \& Perrett, D. I. (in press). Attraction to men and women predicts sexual dimorphism preferences. International Journal of Sexual Health.

DeBruine, L. M. (2018). debruine/webmorph: Beta release 2 (Version v0.0.0.9001). Zenodo. http://doi.org/10.5281/zenodo.1162670

DeBruine, L. M. (2019). Experimentum: Beta release 1 (Version v.0.1). Zenodo. http://doi.org/10.5281/zenodo.2634356

DeBruine, L. M. \& Jones, B. C. (2017): Young adult white faces with manipulated versions. Figshare.

Holzleitner, I. J., Lee, A. J., Hahn, A., Kandrik, M., Bovet, J., Renoult, J. P., ... Jones, B. C. (in press). Comparing theory-driven and data-driven attractiveness models using images of real women's faces. Journal of Experimental; Psychology: Human Perception and Performance. 
Hong, G., Luo, M. R. and Rhodes, P. A. (2001). A study of digital camera colorimetric characterization based on polynomial modelling. Color Research and Application, 26, 76-84.

Jones, B. C., Hahn, A. C., Fisher, C. I., Wang, H., Kandrik, M., Han, C., ... DeBruine, L. M. (2018). No compelling evidence that preferences for facial masculinity track changes in women's hormonal status. Psychological Science, 29, 996-1005.

Kandrik, M., \& DeBruine, L. M. (2012). Self-rated attractiveness predicts preferences for opposite-sex faces, while self-rated sex-typicality predicts preferences for same-sex faces. Journal of Evolutionary Psychology, 10, 177186.

Kuznetsova, A., Brockhoff, P. B., \& Christensen, R. H. B. (2015). ImerTest: Tests for random and fixed effects for linear mexed effect models. Retrieved from https://CRAN.R-project.org/package=ImerTest

Little, A. C., \& Mannion, H. (2006). Viewing attractive or unattractive same-sex individuals changes self-rated attractiveness and face preferences in women. Animal Behaviour, 72, 981-987.

Little, A. C., Jones, B. C., Penton-Voak, I. S., Burt, D. M., \& Perrett, D. I. (2002). Partnership status and the temporal context of relationships influence human female preferences for sexual dimorphism in male face shape. Proceedings of the Royal Society of London, B, 269, 1095-1100.

Little, A. C., Burt, D. M., Penton-Voak, I. S., \& Perrett, D. I. (2001). Selfperceived attractiveness influences human female preferences for sexual dimorphism and symmetry in male faces. Proceedings of the Royal Society B: Biological Sciences, 268, 39-44. 
O’Connor, J. J., Feinberg, D. R., Fraccaro, P. J., Borak, D. J., Tigue, C. C., Re, D. E., ... \& Tiddeman, B. (2012). Female preferences for male vocal and facial masculinity in videos. Ethology, 118, 321-330.

Penton-Voak, I. S., Little, A. C., Jones, B. C., Burt, D. M., Tiddeman, B. P., \& Perrett, D. I. (2003). Female condition influences preferences for sexual dimorphism in faces of male humans (Homo sapiens). Journal of Comparative Psychology, 117, 264-271

R Core Team. (2013). A language and environmental for statistical computing. Vienna, Austria: R Foundation for Statistical Computing.

Simonsohn, U. (2015). Small Telescopes: Detectability and the Evaluation of Replication Results. Psychological Science, 26, 559-569.

Smith, F. G., Jones, B. C., Welling, L. L., Little, A. C., Vukovic, J., Main, J. C., \& DeBruine, L. M. (2009). Waist-hip ratio predicts women's preferences for masculine male faces, but not perceptions of men's trustworthiness.

Personality and Individual Differences, 47, 476-480.

Vukovic, J., Feinberg, D. R., Jones, B. C., DeBruine, L. M., Welling, L. L., Little, A. C., \& Smith, F. G. (2008). Self-rated attractiveness predicts individual differences in women's preferences for masculine men's voices. Personality and Individual Differences, 45, 451-456.

Zietsch, B. P., Lee, A. J., Sherlock, J. M., \& Jern, P. (2015). Variation in women's preferences regarding male facial masculinity is better explained by genetic differences than by previously identified context-dependent effects. Psychological Science, 1-9. 